\title{
Estimation of the retinal nerve fibre layer thickness in the papillomacular area of long standing stage IV macular holes
}

Alex Assi, Patrick Watts, James McAllister

\begin{abstract}
Aim-To compare the thickness of the retinal nerve fibre layer (RNFL) in the papillomacular area of patients with long standing stage IV macular holes with age matched controls, using a scanning laser polarimeter.

Methods-The nerve fibre analyser (NFA) was used to measure the mean thickness of the RNFL around the optic nerve head, the thickness values of temporal and nasal 45 degrees sectors and the integral values in $\mathbf{1 0}$ patients with macular holes and in $\mathbf{1 0}$ age matched controls.

Results-The mean RNFL thickness around the optic nerve head was 79.71 (SD 15.06) $\mu \mathrm{m}$ in the macular hole group and $75.1(10.8) \mu \mathrm{m}$ in the control group ( $\mathrm{p}=$ 0.44). The mean thickness in the temporal sector was $63.69(12.08) \mu \mathrm{m}$ in the macular hole group and $58.65(8.9) \mu \mathrm{m}$ in the control group $(p=0.3)$. The mean ratio between the temporal and nasal sector thickness values was 0.8441 in the macular hole group and 0.7819 in the controls $(p=0.42)$.

Conclusions-There was no significant difference in the thickness of the RNFL in the papillomacular area in the two groups. This suggests that there may be no changes in the thickness of the RNFL in patients with long standing macular holes. (Br F Ophthalmol 1999;83:573-576)
\end{abstract}

Recent developments in vitreoretinal surgical techniques have widened the indications and treatment modalities and improved the prognosis of macular holes. Several studies have demonstrated the efficacy of vitrectomy in the treatment of macular holes and the results seem more favourable in cases of newly formed ones. ${ }^{1-3}$ Reports suggest a poor surgical prognosis in long standing macular holes, possibly due to photoreceptor degeneration. ${ }^{1}$ Frangieh et al provided histopathological evidence of photoreceptor degeneration extending from the edge in stage III macular holes with associated cystoid degeneration in the outer plexiform and inner nuclear layers of the retina. ${ }^{4}$

Hence in vivo investigation of the retinal nerve fibre layer (RNFL) thickness in the papillomacular area of patients with long standing stage IV macular holes may provide evidence of secondary changes affecting the retinal nerve fibres and may therefore explain the poorer visual results following surgical treatment in this group of patients. Several techniques like red-free fundus ophthalmos- copy, optical coherence tomography, ${ }^{5}$ scanning laser tomography, ${ }^{6}$ scanning laser microperimetry, ${ }^{7}$ laser biomicroscopy, ${ }^{8}$ and photography can be used to visualise and evaluate subjectively qualitative changes in the peripapillary nerve fibre layer. On the other hand the scanning laser polarimeter can quantitatively measure the RNFL thickness. ${ }^{9}$ This is indirectly estimated in vivo depending on the amount of polarisation change induced by the RNFL on a polarised laser beam passing through it. ${ }^{10}$ Although the nerve fibre analyser (NFA) has been used to demonstrate nerve fibre layer defects after macular hole surgery, ${ }^{11}$ no study has yet evaluated its role in estimating the RNFL thickness in the papillomacular area of long standing stage IV macular holes.

The aim of this study was to compare the RNFL thickness in the papillomacular area of patients with long standing stage IV macular holes and age matched normal controls using the scanning laser polarimeter.

\section{Materials and methods}

The NFA version 2.1.00 (Laser Diagnostic Technologies Inc, San Diego, CA, USA) was used to measure in vivo the thickness of the RNFL. It consists of a laser source, a polariser, a scanning unit, a polarisation modulator, a compensator, and a polarisation detector. When a polarised near infrared light $(780 \mathrm{~nm})$ is projected across the ocular media to the retina, it double passes the RNFL before emerging from the eye. The parallel microtubules within the retinal ganglion cell axons have a diameter smaller than the wavelength of the polarised laser light and are thought to induce a change in its state of polarisation. The RNFL behaves as a birefringent medium and changes the state of polarisation of the light passing through, which is detected and quantified by the NFA. The amount of polarisation change (retardation) in the light reflected back from the retina correlates with the RNFL thickness. In postmortem monkey eyes, Weinreb and associates found that one degree of retardation corresponded to a RNFL thickness of approximately $7.4 \mu \mathrm{m} .{ }^{12}$ The compensator corrects for the effect of lens and corneal birefringence on the RNFL retardation measurements.

A complete scan covers $256 \times 256$ individual retinal positions (pixels) in 0.7 seconds. The computer algorithm then forms a colour coded retardation map by calculating the amount of retardation in degrees for each pixel within the field of view. Thus, retardation is a measure of relative, not absolute, RNFL thickness. 


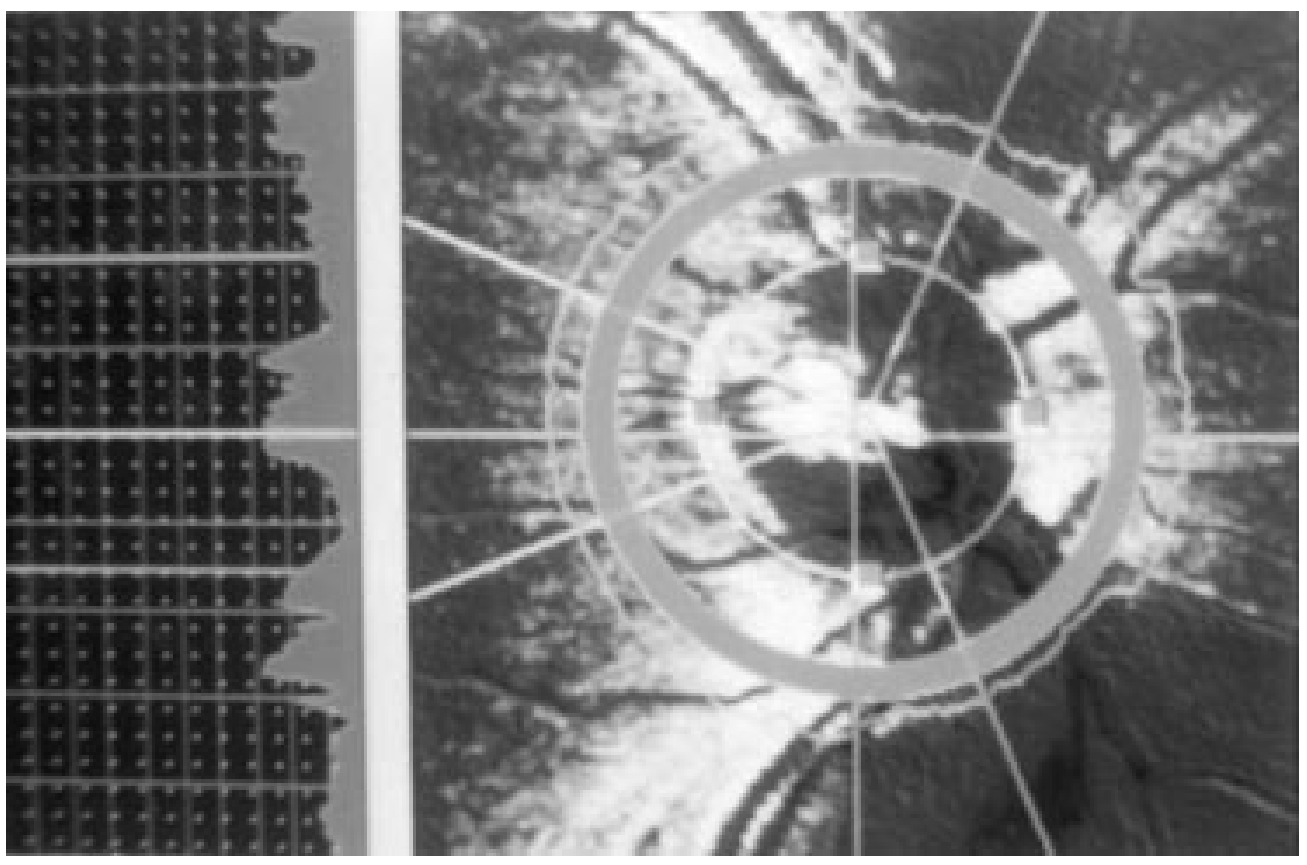

Figure 1 Retardation map of the retinal nerve fibre layer (RNFL) thickness of the right eye of a patient with macular hole. The RNFL thickness is measured over a circular band of 10 pixels width at a distance of 1.5 disc diameter around the optic disc. The temporal 45 degree sector of this band corresponds to the region of the papillomacular bundle.

We have recruited for this study 10 patients with long standing stage IV macular holes as defined by Gass, ${ }^{13}$ diagnosed and documented for at least 1 year (average 3.0 (SD 1.9) years). Controls were randomly selected from the same residential postcode and matched for age, sex, and the side of the affected eye.

Inclusion criteria included the absence of any other known ocular pathology affecting the macula, retina, or optic nerve. Exclusion criteria included diabetes, glaucoma, ocular hypertension, positive family history of glaucoma, refractive error exceeding plus or minus 4 dioptres, and abnormal appearance of the optic disc, peripapillary area, and macula (except for the hole) at the time of the examination.

In order to minimise the subjective effects of operator bias in selecting the overall intensity and illumination, all the images were acquired by one of the authors (PW) with considerable experience using this machine (NFA version 2.1.00 Laser Diagnostic Technologies Inc, San Diego, CA, USA). Informed consent was obtained before imaging and for each subject six images were taken through undilated pupils in order to improve the accuracy of our measurements. Details of image acquisition have been reported elsewhere. ${ }^{14}$

A 15 degree field size was used and the optic nerve head was centred and focused in the middle of the monitor LCD display. The RNFL thickness was measured over a circular band of 10 pixels width at a distance of 1.5 disc diameter around the optic disc. The band centred around the optic nerve head was divided into four sectors: temporal and nasal 45 degrees each and superior and inferior 135 degrees. The software calculates the average nerve fibre layer retardation value along the entire circle and in the four sectors and displays it on the screen (Fig 1). The integral under the curve is also calculated (polarimetric data analysis) to represent the cross sectional area of nerve fibre tissue along the circle at the selected distance from the disc margin. The values obtained in the temporal sector reflect best the thickness of the RNFL in the papillomacular area.

The retardation values of the best three images acquired were averaged. The NFA measured the mean RNFL thickness within a 10 pixel width circular band. The mean RNFL thickness values in the four sectors, integral values of the RNFL thickness and ratios between the sectors were calculated and used for statistical analysis for each subject in the two groups of patients.

We used a Student's $t$ test to compare the thickness of the RNFL in patients with macular holes and matched controls. A p value of less than 0.05 was considered statistically significant.

\section{Results}

Patients with macular holes and controls were all white. The female:male ratio was 2.33:1 with an average age of 73.1 (SD 7.92) years (range 58-84 years). The average best corrected visual acuity of the examined eye was $6 / 60$ (Snellen) in the macular hole group and $6 / 6$ in the control group.

The total (peripapillary) thickness of the RNFL varied from a minimum of $51.2 \mu \mathrm{m}$ to a maximum of $101.9 \mu \mathrm{m}$ with a mean of 79.71 (15.06) $\mu \mathrm{m}$ in the macular hole group and from a minimum of $57.1 \mu \mathrm{m}$ to a maximum of $89.1 \mu \mathrm{m}$ with a mean of $75.1(10.8) \mu \mathrm{m}$ in the control group showing no statistical significant difference between the two groups $(p=0.44)$. There was also no statistical significant difference between the mean total polar integral of the macular hole group $\left(0.6774 \mathrm{~mm}^{2}\right)$ and that of the control group $\left(0.6606 \mathrm{~mm}^{2}\right)(\mathrm{p}=0.77)$ 
Table 1 Retinal nerve fibre layer thickness (RNFL) thickness and integral values in patients with macular holes and in healthy subjects

\begin{tabular}{llll}
\hline & Macular holes & Controls & p Value \\
\hline Mean total thickness of the RNFL $(\mu \mathrm{m})(\mathrm{SD})$ & $79.71(15.06)$ & $75.1(10.8)$ & 0.44 \\
Mean total polar integral $\left(\mathrm{mm}^{2}\right)$ & 0.6774 & 0.6606 & 0.77 \\
Mean temporal thickness of the RNFL $(\mu \mathrm{m})(\mathrm{SD})$ & $63.69(12.08)$ & $58.65(8.9)$ & 0.3 \\
Mean temporal integral $\left(\mathrm{mm}^{2}\right)$ & 0.0687 & 0.0665 & 0.72 \\
Mean ratio of temporal $/$ nasal thickness & 0.8441 & 0.7819 & 0.42 \\
Mean ratio of temporal/nasal integral & 0.2906 & 0.2694 & 0.43 \\
\hline
\end{tabular}

The mean thickness of the RNFL thickness in the 45 degree temporal sector in the macular hole group was 63.69 (12.08) $\mu \mathrm{m}$ with a range of $41.6-79.8 \mu \mathrm{m}$.

The mean thickness in an identical area in the control group was $58.65(8.9) \mu \mathrm{m}$ with a range of $41.7-73.4 \mu \mathrm{m}$. There was no statistical significant difference between the two groups $(\mathrm{p}=0.3)$. There was also no statistical significant difference between the mean temporal integral of the macular hole group $(0.0687$ $\left.\mathrm{mm}^{2}\right)$ and that of the control group $(0.0665$ $\left.\mathrm{mm}^{2}\right)(\mathrm{p}=0.72)$.

Ratios of the temporal to nasal thickness values had a mean of 0.8441 (range 0.624 $1.236)$ in the macular hole group and a mean of 0.7819 (range $0.631-1.081$ ) in the control group showing no statistical significant difference between the two groups $(p=0.42)$. There was also no statistical significant difference between the mean ratio of the temporal to nasal integral of the macular hole group (0.2906) and that of the control group $(0.2694)(p=0.43)($ Table 1$)$.

\section{Discussion}

Evaluation of the RNFL in vivo has been so far limited to qualitative or semiquantitative assessments of red-free fundus photographs ${ }^{15} 16$ or images obtained with the scanning laser ophthalmoscope ${ }^{17}$ optical coherence tomography, ${ }^{5}$ and scanning laser tomography. ${ }^{6}$ Scanning laser polarimetry is a new method for measuring NFL thickness in vivo with the advantages of not relying on optical imaging principles (therefore avoiding refractive errors) and not requiring a reference plane. It also obviates the need for pupillary dilatation and remains reliable in the presence of mild lens opacities.

Several studies have investigated the variation of NFL thickness measurements using scanning laser polarimetry in normal subjects and in patients with ocular diseases. These studies suggest a progressive decay of ganglion fibres with age, ${ }^{18} 19$ a reduction of the NFL thickness in patients with glaucoma ${ }^{20}$ and ocular hypertension, ${ }^{21}$ and a difference in thickness between whites and Afro-Caribbeans. ${ }^{18}$ These results are in overall agreement with other clinical and histological studies which validate the technique of scanning laser polarimetry as one of the first that can quantitatively determine NFL thickness in living humans.

Using the scanning laser polarimeter we intended to investigate any difference in the thickness of the RNFL in the papillomacular area between patients with long standing stage IV macular holes and normal controls. Our results show no statistical significant difference in the mean total thickness and the mean total polar integral of the RNFL between the two groups of patients. Similarly, there was no statistically significant difference in the mean thickness or in the mean integral of the RNFL in the 45 degree temporal sector between the two groups of patients. Using ratios of the measured values to improve intraobserver reproducibility ${ }^{14}$ we also find no statistical significant difference in the mean ratio of the thickness measurements and integral values of the temporal to nasal sector between the two groups. We used the temporal to nasal ratio calculations in particular as retardation measurements using scanning laser polarimetry were shown to be least variable in the nasal and temporal than in the superior and inferior sectors. ${ }^{2022} 23$

In previous studies the NFA was able to associate RNFL defects to corresponding perimetric visual field loss in patients with glaucoma ${ }^{9}$ and in patients with visual field defects following macular hole surgery. ${ }^{11}$ Moreover, lower readings have been reported in ocular hypertensive patients compared with healthy controls. ${ }^{24}$ However, ocular hypertensives who progressed to visual field loss and corresponding RNFL defects were never shown to have initial demonstrable quantitative changes in their RNFL before visual field loss supervened. It is therefore possible that the NFA cannot pick up very early localised changes in the RNFL thickness. This may explain the absence of detectable significant changes in the papillomacular area of our patients where one might expect localised ganglion nerve fibre atrophy secondary to photoreceptor degeneration at the edge of the macular hole and associated cystoid degeneration in the outer plexiform and inner nuclear layers of the retina. ${ }^{4}$ Conversely, it may also be that limited and localised photoreceptor damage during the process of macular hole formation and the ensuing months may not induce any changes in the thickness of the RNFL in the papillomacular area, hence our results.

On the other hand, our figures show a trend for higher readings (though not statistically significant) in the total and sector thickness values of the RNFL in the macular hole group, where one would expect lower values, if photoreceptor degeneration is to induce a reduction in the RNFL thickness. This paradox could be explained by a subclinical extension of intraretinal fluid towards the disc and into the nerve fibre layer from the edge of the macular hole. ${ }^{25}$ On the other hand vitreous traction causing elevation of Henle's fibre layer $^{26}$ could also be responsible for the mechanical bunching up of retinal nerve fibres during formation of the hole. The hypothetical mechanical distortion in the architecture of the photoreceptors and the corresponding RNFL across the retinal thickness in the papillomacular area may sometimes occur in the absence of cellular degeneration. This could therefore explain the occasional good visual results in some chronic macular holes despite late surgery. ${ }^{27} 28$ 
In conclusion, we found no significant changes in the thickness of the RNFL of patients with long standing full thickness macular holes. However, our number of cases involved is small to draw definite conclusions about the preservation of neural pathways. Future larger studies may allow the NFA to triage such cases for surgical treatment.

1 Ryan EH, Gilbert HD. Results of surgical treatment of recent-onset full thickness idiopathic macular holes. Arch Ophthalmol 1994;112:1545-53.

2 Willis AW, Garcia-Cosio JF. Macular hole surgery. Comparison of long-standing versus recent macular holes. $O p h-$

thalmology 1996;1033:1811-14.
3 Wendel RT, Patel AC, Kelly NE, et al. Vitreous surgery for macular holes. Ophthalmology 1993;100:1671-6.

4 Frangieh GT, Green WR, Engel HM. A histopathological study of macular cysts and holes. Retina 1981;1:311-36.

5 Hee MR, Pulifato CA, Wong C, et al. Optical coherence Hee MR, Pulifato CA, Wong C, et al. Optical coherence
tomography of macular holes. Ophthalmology 1995;102: tomogra $748-56$.

6 Hudson C, Charles SJ, Flanagan JG, et al. Objective morphological assessment of macular hole surgery by scanning laser tomography. Br F Ophthalmol 1997;81:10716.

7 Sjaarda RN, Frank DA, Glaser BM, et al. Assessment of vision in idiopathic macular holes with macular microperimetry using the scanning laser ophthalmoscope. Ophthalmology 1993;100:1513-18

8 Kiryu J, Shahidi M, Ogura Y, et al. Illustration of the stages of idiopathic macular holes by laser biomicroscopy. Arch Ophthalmol 1995;113:1156-60.

9 Weinreb RN, Shakiba S, Sample PA, et al. Association between quantitative nerve fiber layer measurement and visual field loss in glaucoma. Am $\mathcal{F}$ Ophthalmol 1995;120: 732-8.

10 Dreher AW, Reiter K, Wienreg R. Spatially resolved birefringence of retinal nerve fiber layer assessed with retinal laser ellipsometer. Appl Optics 1992;31:3730-5.

11 Hutton WL, Fuller DG, Snyder WB, et al. Visual field defects after macular hole surgery. Ophthalmology 1996; 103:2152-9

12 Weinreb RN, Dreher AW, Coleman A, et al. Histopathologic validation of Fourier-ellipsometry measurements of retinal nerve fiber layer thickness. Arch Ophthalmol 1990;108:55760 .
13 Gass JDM. Reappraisal of biomicroscopic classification of stages of development of a macular hole. Am $\mathcal{F}$ Ophthalmol 1995;119:752-9.

14 Junghardt A, Schmid MK, Schipper I, et al. Reproducibility of the data determined by scanning laser polarimetry. Graefes Arch Clin Exp Ophthalmol 1996;234;628-32.

15 Airaksinen PJ, Drance SM, Douglas GR, et al. Diffuse and localized nerve fiber loss in glaucoma. Am 7 Ophthalmol 1984;98:566-71.

16 Quigley HA, Reacher M, Katz, et al. Quantitative grading of nerve fiber layer photography. Ophthalmology 1993;100: 1800-7.

17 Miglior S, Rossetti L, Brigatti L, et al. Reproducibility of retinal nerve fiber layer evaluation by dynamic scanning laser ophthalmoscope. Am f Ophthalmol 1994;118:16-23.

18 Poinoosawmy D, Fontana L, Wu JX, et al. Variation of nerve fiber layer thickness measurements with age and ethnicity by scanning laser polarimetry. $\mathrm{Br} \mathcal{F}$ Ophthalmol 1997;81: 350-4.

19 Chi QM, Tomita G, Inazumi K, et al. Evaluation of the effect of aging on the retinal nerve fiber layer thickness using scanning laser polarimetry. F Glaucoma 1995;4:406-13.

20 Weinreb RN, Shakiba S, Zangwill L. Scanning laser polarimetry to measure the nerve fiber layer of normal and glaucomatous eyes. Am f Ophthalmol 1995;119:627-36.

21 Tjo-Fo-Sang MJ, De Vries J, Lemij HG. Measurement by nerve fiber analyzer of retinal nerve fiber layer thickness in normal subjects and patients with ocular hypertension. $\mathrm{Am}$ f Ophthalmol 1996;122:220-7.

22 Caprioli J. The contour of the juxtapapillary nerve fiber layer in glaucoma. Ophthalmology 1990;97:358-66.

23 Caprioli J, Miller JM. Measurement of relative nerve fiber layer surface height in glaucoma. Ophthalmology 1989;96: 633-41.

24 Anton A, Zangwill L, Emdadi A, et al. Nerve fiber layer measurements with scanning laser polarimetry in ocular hypertension. Arch Ophthalmol 1997;115:331-4.

25 Asrani S, Zeimer R, Goldberg MF, et al. Serial optical sectioning of macular holes at different stages of development. Ophthalmology 1998;105:66-77.

26 Kishi S, Kamei Y, Shimizu K. Tractional elevation of Henle's fiber layer in idiopathic macular holes. Am f Ophthalmol 1995;120:486-96.

27 Kokame GT. Macular hole surgery for chronic macular holes. Retina 1996;16:75-8.

28 Thompson JT, Sjaarda RN, Lansing MB. The results of vitreous surgery for chronic macular holes. Retina 1997;17: 493-501. 